\title{
INTERVAL SENSITIVITY ANALYSIS OF DYNAMIC RESPONSE ENVELOPES FOR UNCERTAIN MECHANICAL STRUCTURES
}

\author{
David Moens $^{1,2}$, Dirk Vandepitte ${ }^{1}$ \\ ${ }^{1}$ K.U.Leuven, Dept. Mechanical Engineering, div. PMA \\ Celestijnenlaan 300B, B-3001 Heverlee, Belgium \\ david.moens@mech.kuleuven.be, dirk.vandepitte@mech.kuleuven.be \\ ${ }^{2}$ Postdoctoral research fellow of the Research Foundation - Flanders
}

Keywords: Interval finite element analysis, fuzzy finite elements, envelope FRF

\begin{abstract}
This paper introduces an interval sensitivity procedure that calculates the sensitivity of the envelope response function in the outcome of the interval FRF analysis to each individual interval model uncertainty. The procedure focuses on the calculation of the sensitivity of the bounds defining the FRF response range to the width of each individual uncertain input parameter. The approach differs from the classical sensitivity analysis in the fact that it does not calculate local changes on the output resulting from local changes in the input. The interval sensitivity result describes the change of the response interval width, taking into account a change in the parameter interval width. The paper first introduces the concept of interval sensitivities. It then describes the theoretical background of the interval FRF procedure, on which the interval sensitivity analysis is applied. Finally, the method is illustrated on a numerical example.
\end{abstract}




\section{INTRODUCTION}

Non-deterministic approaches are gaining momentum in the field of finite element analysis. The ability to include non-deterministic properties is of great value for a design engineer. It enables realistic reliability assessment that incorporates the uncertain aspects of the design. Furthermore, the design can be optimised for robust behaviour under varying external influences. Recently, criticism arises on the general application of the probabilistic concept in this context. Especially when objective information on the uncertainties is limited, the subjective probabilistic analysis result proves to be of little value, and does not justify its high computational cost. Consequently, alternative non-probabilistic concepts are used for non-deterministic finite element analysis, as e.g. the fuzzy and interval concept.

Recently, a fuzzy finite element methodology to calculate a fuzzy frequency response function (FRF) of uncertain undamped structures was developed by the authors [1]. The procedure consists of the solution of a sequence of interval problems. The goal of the interval analysis is to calculate the envelope of the FRF taking into account that the input uncertainties can vary within the bounded space defined by their combined intervals. For this purpose, a hybrid procedure involving both a global optimisation step and an interval arithmetic step has been developed. The resulting envelope response function gives a clear view on the possible variation of the response in the frequency domain.

The applicability of this interval response analysis was proven on realistic case studies [2, 3]. In this result, all uncertain parameters are considered to act simultaneously. While this is often the most realistic representation of the physical condition of the actual product, for design purposes, it can be of great value to know the contribution of the individual uncertainties to the response range obtained from the interval analysis. This enables a designer to distinct between the non-deterministic influences that have an important contribution to the fuzziness on the dynamic behaviour of the design, and those that have little or no influence. This distinction can be very valuable in the definition of e.g. tight design tolerances or realistic allowable working conditions, and as such, could lead to less conservative designs.

In this context, this paper introduces a methodology to calculate the sensitivity of the envelope response function in the outcome of the interval FRF analysis to each individual interval model uncertainty. The principal goal of this approach is to gain knowledge in the behaviour of the response boundary curves as determined with the interval procedure with repect to changes in the parameter intervals in the model. For this purpose, a new concept called interval sensitivity analysis is introduced. Interval sensitivities represent the rate of change of the width of the range of an output component of an interval analysis when the width of an input parameter changes. This is the fundamental difference between the proposed interval sensitivities and the classical numerical sensitivity studies which always focus on a local sensitivity. These local perturbations are generally not valid over the range of an interval. Therefore, this new concept of interval sensitivities is introduced.

Section 2 introduces the principal idea of the interval sensitivities from a mathematical point of view, and discusses how these interval sensitivities can be calculated. Section 3 then briefly summarises the algorithm behind the interval response analysis. Section 4 shows how the interval sensitivity concept can be applied to calculate response range sensitivities. Finally, section 5 illustrates the approach on a realistic case study. 


\section{INTERVAL SENSITIVITIES}

The principal idea behind the interval sensitivities is that they should quantify the impact of an individual interval parameter at the input of the problem on the interval occurring on the numerical result of the interval analysis. This means that they represent the degree of influence of the width of each input interval on the width of the output interval. Therefore, a new analysis procedure is introduced. Section 2.1 first gives a brief basic introduction of numerical interval analysis. Then, section 2.2 introduces the general concept of the interval sensitivities, while section 2.3 focuses on their calculation in the context of a numerical interval analysis.

\subsection{Numerical interval analysis}

In order to introduce the concept of interval sensitivities in the context of general numerical interval analysis, a basic problem is analysed. The considered interval problem consists of the calulation of the range of the result of a function $f$ applied on a single parameter $p$. The relationship between input $p$ and output $y$ is expressed as:

$$
y=f(p)
$$

The aim of general interval analysis is to calculate the range of the output $y$ of this function taking into account that the parameter $p$ can vary within the range between its lower bound $\underline{p}$ and upper bound $\bar{p}$. This is expressed as:

$$
y^{I}=f^{I}\left(p^{I}\right)
$$

with

$$
\begin{aligned}
& p^{I}=[\underline{p}, \bar{p}] \\
& y^{I}=[\underline{y}, \bar{y}]
\end{aligned}
$$

and $f^{I}$ the interval function that represents the relationship between the input interval and the output interval. Two basis strategies are commonly used to calculate the resulting interval $y^{I}$, i.e. the global optimisation approach, and the interval arithmetic approach:

- In the global optimisation approach, the interval result is calculated by minimising and maximising the function $f$ over the domain $p^{I}$ of the uncertain parameter $p$ :

$$
\begin{aligned}
\underline{y} & =\min _{p \in p^{I}} f(p) \\
\bar{y} & =\max _{p \in p^{I}} f(p)
\end{aligned}
$$

This strategy gives the exact range of the output value. The computational cost however can be high, especially when complex functions are analysed, or when the number of uncertain parameters increases.

- The interval arithmetic approach approximates the exact range of the output value. In this approach, the function $f$ is implemented as a sequence of basic operations $(+,-, \times, \div)$. For these basic operation, an interval arithmetic equivalent exists:

$$
\begin{aligned}
a^{I}+b^{I} & =[\underline{a}+\underline{b}, \bar{a}+\bar{b}] \\
a^{I}-b^{I} & =[\underline{a}-\bar{b}, \bar{a}-\underline{b}] \\
a^{I} \times b^{I} & =[\min (\underline{a} \times \underline{b}, \underline{a} \times \bar{b}, \bar{a} \times \underline{b}, \bar{a} \times \bar{b}), \max (\underline{a} \times \underline{b}, \underline{a} \times \bar{b}, \bar{a} \times \underline{b}, \bar{a} \times \bar{b})] \\
a^{I} / b^{I} & =a^{I} \times\left[\frac{1}{\bar{b}}, \frac{1}{\underline{b}}\right], \text { if } 0 \notin b^{I}
\end{aligned}
$$


By applying these interval equivalents on each deterministic operation of the original function $f$, the output range is approximated. This is a straightforward and computationally inexpensive approach that can be applied on any function that can be expressed as a sequence of basic operations. However, it suffers from an important drawback, i.e. the high degree of conservatism in the final result (see e.g. [4] for the discussion on the application of this approach for interval finite element analysis).

The procedure for the calculation of envelope responses utilises both these approaches in a hybrid procedure, as discussed in section 3 .

\subsection{General concept of interval sensitivities}

In order to introduce the concept of the interval sensitivities, the relationship between the input and output interval is now expressed through the function $f^{\Delta}$ which relates the input interval radius to the output interval radius. The interval radius is defined as:

$$
\Delta y=\frac{\bar{y}-\underline{y}}{2}
$$

Similar, the interval midpoint equals:

$$
y^{C}=\frac{\bar{y}+\underline{y}}{2}
$$

The relationship between the input and output intervals is written as:

$$
\Delta y=f^{\Delta}(\Delta p)
$$

This interval function $f^{\Delta}$ is the basis for the definition of the interval sensitivities. The interval sensitivity of the output interval $y^{I}$ with respect to the input interval $p^{I}$ is defined as:

$$
\delta_{p^{I}}^{y^{I}}=\frac{\partial(\Delta y)}{\partial(\Delta p)}=\frac{\partial f^{\Delta}(\Delta p)}{\partial(\Delta p)}
$$

The interval sensitivity as defined in this equation expresses the relationship between the change in absolute interval widths on the input and the output side of the problem.

\subsection{Calculation of interval sensitivities}

Based on their definition, it is clear that the interval sensitivities can be calculated if the relationship between the radius of input and output parameters is known, expressed as the interval function $f^{\Delta}$ in equation $(13$. In general, this relationship cannot be derived directly. However, from the definition of the interval radius in equation (11), the absolute interval sensitivity is obtained as:

$$
\begin{aligned}
\delta_{p^{I}}^{y^{I}} & =\frac{\partial(\Delta y)}{\partial(\Delta p)} \\
& =\frac{1}{2}\left(\frac{\partial \bar{y}}{\partial(\Delta p)}-\frac{\partial \underline{y}}{\partial(\Delta p)}\right) \\
& =\frac{1}{2}\left(\delta_{p^{I}}^{\bar{y}}-\delta_{p^{I}}^{y}\right)
\end{aligned}
$$


This last equation defines the upper and lower bound sensitivities $\delta_{p^{I}}^{\bar{y}}$ and $\delta_{p^{I}}^{\underline{y}}$. Similar to the interval sensitivities themselves, these represent the rate of change of the lower and upper bound on the result of an interval analysis with respect to a change in the input parameter interval width. Since both the optimisation and the interval arithmetic approach are present in the procedure for envelope response function calculations, sections 2.3.1 and 2.3.2 now focus on the calculation of these interval bound sensitivities in the context of these respective approaches.

\subsubsection{Interval sensitivities in the global optimisation approach}

Consider the simple unidimensional interval problem as defined in section 2.1 and the global optimisation solution strategy. The lower and upper bound sensitivities express the change in the result interval upper bound $\bar{y}$ and lower bound $\underline{y}$ with respect to a change in the input parameter interval radius $\Delta p$. Three important observations are made:

- Since the bounds $y$ and $\bar{y}$ are found by evaluating the function $f$ at parameter values within the interval $p^{I}$, the interval width of $y^{I}$ cannot decrease when the input interval width $\Delta p$ increases. This means for the change in the lower and upper bound that:

$$
\begin{aligned}
& \delta_{p^{I}}^{\frac{y}{y}}=\frac{\partial \underline{y}}{\partial(\Delta p)} \leq 0 \\
& \delta_{p^{I}}^{\bar{y}}=\frac{\partial \bar{y}}{\partial(\Delta p)} \geq 0
\end{aligned}
$$

- Also, it can be seen that the output bound sensitivities only equal zero if the respective bounds $\underline{y}$ and $\bar{y}$ are obtained for parameter values $p^{\underline{y}}$ and $p^{\bar{y}}$ that embody local optima of the function $f$ applied on the interval $p^{I}$. Indeed, if an output bound stems from a local optimum in the observed input domain, an incremental increase in the parameter region $\delta(\Delta p)$ does not change the location of this bound in the interval. Consequently, the bound is insensitive to a change in the input interval width.

- If an output bound does not stem from a local optimum, this means that it is located on either of the vertex point. This means that $p^{\underline{y}}=\underline{p} \vee \bar{p}$ or $p^{\bar{y}}=\underline{p} \vee \bar{p}$. Since the input locations do not represent a local optimum, it is easily shown that the output bound remains on the same vertex location if an incremental change is applied on the input interval. Furthermore, the derivative of the output value $y$ with respect to the input parameter $p$ at this vertex point is not zero. Actually, this derivative expresses the rate of change of the output value at this vertex point with respect to a change in the input parameter $p$. Consequently, this derivative also represents this bound's interval sensitivity.

Based on these observations, it is concluded that the upper and lower bound interval sensitivities are directly related to the behaviour of the analysed function $f$ in the input parameter locations $p \underline{y}$ and $p^{\bar{y}}$ where the output bounds are obtained. In summary, the lower and upper bound interval sensitivities yield:

$$
\begin{aligned}
& \delta_{p^{I}}^{\frac{y}{y}}=-\left|\left(\frac{\partial y}{\partial p}\right)_{p^{\underline{y}}}\right| \\
& \delta_{p^{I}}^{\bar{y}}=\left|\left(\frac{\partial y}{\partial p}\right)_{p^{\bar{y}}}\right|
\end{aligned}
$$


This means that in case the output interval is obtained through a global optimisation procedure, the lower and upper bound sensitivities of the interval result are obtained directly from the gradients in the end points of the optimisation. Finally, the inerval sensitivities of the output interval are obtained by applying equation (17).

\subsubsection{Interval sensitivities in the interval arithmetic approach}

The basic operations in the interval arithmetic approach as defined in equations (7) to (10) state that the bounds on the result of a basic numerical operation are found as a combination of the bounds of the operands. In general, in an interval arithmetic context, the bounds on the interval result are expressed as a function of lower and upper bounds on $n$ operands $q_{i}$. This can be formulated generally using the appropriate functions $g_{l}$ anf $g_{u}$ :

$$
\begin{aligned}
& \underline{y}=g_{l}\left(\underline{q_{1}}, \underline{q_{2}}, \ldots \underline{q_{n}}, \overline{q_{1}}, \overline{q_{2}}, \ldots \overline{q_{n}}\right) \\
& \bar{y}=g_{u}\left(\underline{q_{1}}, \underline{q_{2}}, \ldots \underline{q_{n}}, \overline{q_{1}}, \overline{q_{2}}, \ldots \overline{q_{n}}\right)
\end{aligned}
$$

Based on this expression, the lower bound sensitivity of $y^{I}$ to a parameter $p$ are derived as:

$$
\begin{aligned}
\delta_{p^{I}}^{\underline{y}} & =\frac{\partial \underline{y}}{\partial(\Delta p)} \\
& =\sum_{i=1}^{n} \frac{\partial g_{l}}{\partial \underline{q_{i}}} \frac{\partial \underline{q_{i}}}{\partial(\Delta p)}+\sum_{i=1}^{n} \frac{\partial g_{l}}{\partial \overline{q_{i}}} \frac{\partial \overline{q_{i}}}{\partial(\Delta p)} \\
& =\sum_{i=1}^{n} \frac{\partial g_{l}}{\partial \underline{q_{i}}} \delta_{p^{I}}^{q_{i}}+\sum_{i=1}^{n} \frac{\partial g_{l}}{\partial \overline{q_{i}}} \delta_{p^{I}}^{\overline{q_{i}}}
\end{aligned}
$$

Similar, for the upper bound sensitivity, we have:

$$
\delta_{p^{I}}^{\bar{y}}=\sum_{i=1}^{n} \frac{\partial g_{u}}{\partial \underline{q_{i}}} \delta_{p^{I}}^{\underline{q_{i}}}+\sum_{i=1}^{n} \frac{\partial g_{u}}{\partial \overline{q_{i}}} \delta_{p^{I}}^{\overline{q_{i}}}
$$

This means that in the interval arithmetic approach, the interval sensitivities can be calculated if the bound sensitivities on the operands $q_{i}$ are known. For example, for the interval subtraction, we have:

$$
y=f\left(q_{1}, q_{2}\right)=q_{1}-q_{2}
$$

and thus:

$$
\begin{aligned}
\underline{y} & =\underline{q_{1}}-\overline{q_{2}} \\
\bar{y} & =\overline{q_{1}}-\underline{q_{2}}
\end{aligned}
$$

Based on equations (26) and (27), the bound sensitivities become:

$$
\begin{aligned}
& \delta_{p^{I}}^{\frac{y}{y}}=\delta_{p^{I}}^{\frac{q_{1}}{y^{I}}}-\delta_{p^{I}}^{\overline{q_{2}}} \\
& \delta_{p^{I}}^{\bar{y}}=\delta_{p^{I}}^{\overline{q_{1}}}-\delta_{p^{I}}^{\frac{q_{2}}{p^{I}}}
\end{aligned}
$$

For $p=q_{1}$ or $p=q_{2}$, we have $\delta_{p^{I}}^{\overline{q_{i}}}=1$ and $\delta_{p^{I}}^{q_{i}}=-1$. Substituting this in equations 31, , 32) and 17 , we finally find that the interval sensitivity $\delta_{q_{1} I}^{y^{I}}$ equals one. This is totally in line with the expectation, as there is a linear relationship between the operands $q_{i}$ and the result $y$ of the function.

Section 4 shows how this strategy is applied to calculate the dynamic response range sensitivities in an interval finite element context. 


\section{ALGORITHM FOR ENVELOPE ANALYSIS OF DYNAMIC RESPONSES}

The methodology for the envelope dynamic response analysis as developed by the authors is based on a hybrid interval solution strategy, consisting of a preliminary optimisation step, followed by an interval arithmetic step. In the first part of this procedure, the optimisation is used to translate the interval properties defined on the finite element model to the exact interval modal stiffness and mass parameters of the structure. The calculation of the envelope FRFs in the second part is done by applying the interval arithmetic equivalent of the modal superposition procedure on these interval modal parameters. The final envelope FRFs have been proven to contain only a very limited amount of conservatism. A brief overview of the basic principles of the method is given in this section. The complete mathematical description of the method can be found in Moens et al. [1].

\subsection{The deterministic modal superposition principle}

For undamped structures, the deterministic modal superposition principle states that, considering the first $n_{\text {modes }}$ modes, the frequency response function between degrees of freedom $j$ and $k$ equals:

$$
F R F_{j k}=\sum_{i=1}^{n_{\text {modes }}} F R F_{j k}^{i}=\sum_{i=1}^{n_{\text {modes }}} \frac{\phi_{i_{j}} \phi_{i_{k}}}{{\phi_{\mathbf{i}}}^{T} \mathbf{K} \phi_{\mathbf{i}}-\omega^{2} \phi_{\mathbf{i}}{ }^{T} \mathbf{M} \phi_{\mathbf{i}}}
$$

with $\phi_{\mathbf{i}}$ the $i^{\text {th }}$ eigenvector of the system and $\phi_{i_{j}}$ the $j^{\text {th }}$ component of the $i^{\text {th }}$ eigenvector. Simplification of equation (33) yields:

$$
F R F_{j k}=\sum_{i=1}^{n_{\text {modes }}} \frac{1}{\hat{k}_{i}-\omega^{2} \hat{m}_{i}}
$$

with $\hat{k}_{i}$ and $\hat{m}_{i}$ the modal parameters defined as:

$$
\begin{aligned}
\hat{k}_{i} & =\frac{\phi_{\mathbf{i}}^{T} \mathbf{K} \phi_{\mathbf{i}}}{\phi_{i_{j}} \phi_{i_{k}}} \\
\hat{m}_{i} & =\frac{\phi_{\mathbf{i}}^{T} \mathbf{M} \phi_{\mathbf{i}}}{\phi_{i_{j}} \phi_{i_{k}}}
\end{aligned}
$$

\subsection{Interval finite element FRF analysis}

The modal superposition principle has been translated into an interval finite element method for FRF analysis. Figure 1 gives a graphical overview of the translation of the deterministic algorithm into an interval procedure. On the left-hand side is the deterministic algorithm as described in the previous section. On the right-hand side is the same procedure translated to an equivalent interval algorithm.

The interval method consists of the calculation of the result ranges of the subfunctions appearing in the consecutive steps of the deterministic algorithm. Therefore, the deterministic algorithm is split into three subfunctions. In the first step, step 1.1, the modal stiffness $\hat{k}_{i}$ and mass $\hat{m}_{i}$ are calculated for each considered mode. Step 1.2 then consists of the calculation of the modal FRF contributions $F R F_{j k}^{i}$. Step 1.1 and 1.2 have to be performed for each mode that is taken into consideration in the modal superposition. Therefore, it is referred to as the modal part. In step 2, the superposition is performed by a summation of the modal FRF contributions. 

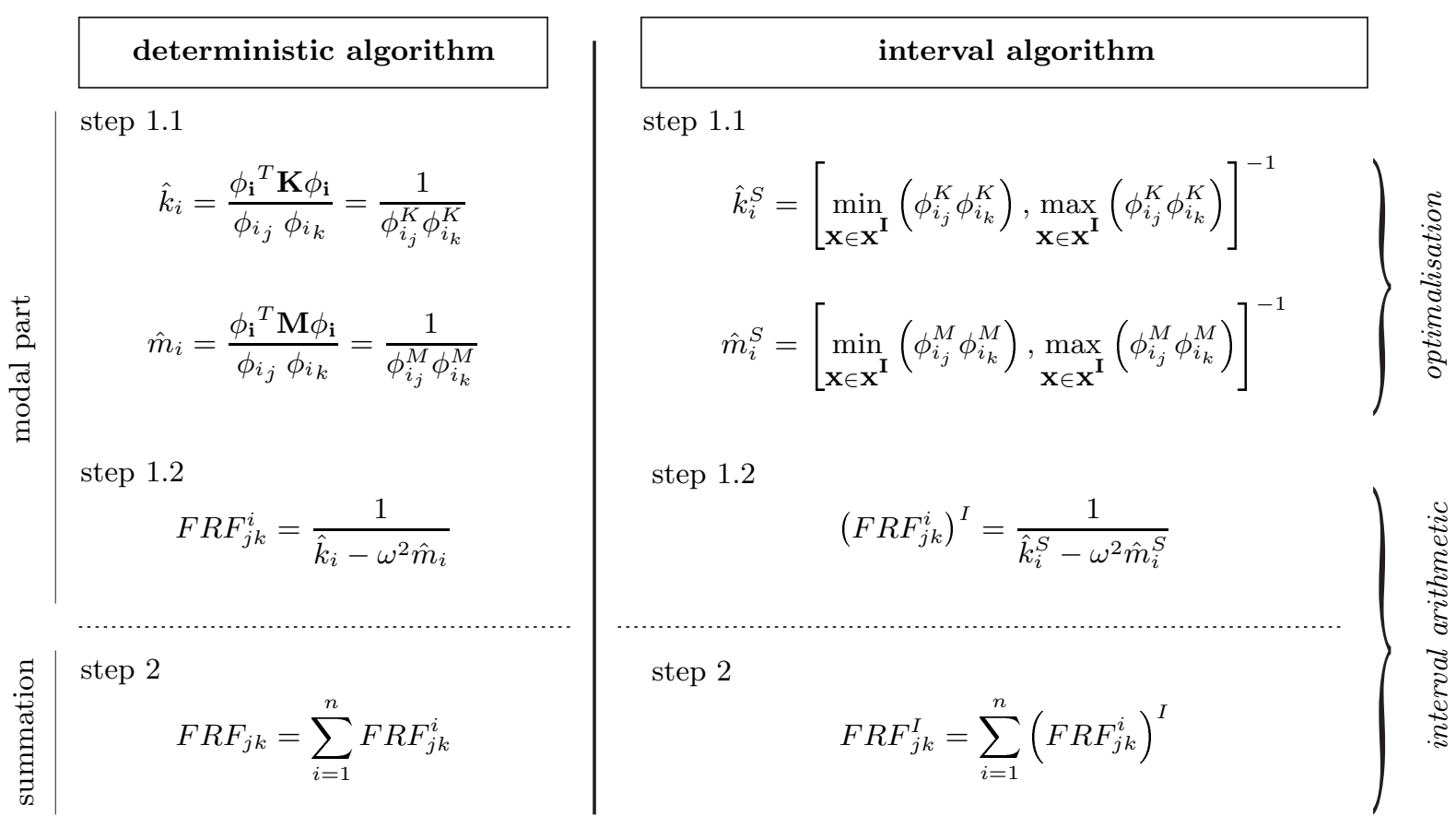

$\begin{array}{lc}\text { step } 1.2 & \left(F R F_{j k}^{i}\right)^{I}=\frac{1}{\hat{k}_{i}^{S}-\omega^{2} \hat{m}_{i}^{S}} \\ \text { step 2 } & F R F_{j k}^{I}=\sum_{i=1}^{n}\left(F R F_{j k}^{i}\right)^{I}\end{array}$

Figure 1: Translation of the deterministic modal superposition algorithm to an equivalent interval procedure

The interval procedure follows the same outline as the deterministic algorithm. Each step now concentrates on the derivation of the range of the subfunctions in the deterministic algorithm:

step 1.1 For all $n_{\text {modes }}$ taken into account, the ranges of possible values that the modal stiffness and mass can adopt have to be determined, taking into account that the uncertain parameters in $\mathrm{x}$ can vary within their respective intervals. These correct ranges of the modal parameters denoted by $\hat{k}_{i}^{S}$ and $\hat{m}_{i}^{S}$ are determined using a minimisation and maximisation over the uncertain interval space $\mathbf{x}^{\mathbf{I}}$. For numerical convenience, the global optimisation is performed on the inverted modal parameters, after which the obtained intervals are inverted in order to obtain the actual modal parameter ranges:

$$
\begin{aligned}
\hat{k}_{i}^{S} & =\left[\min _{\mathbf{X} \in \mathbf{X}^{\mathbf{I}}}\left(\phi_{i_{j}}^{K} \phi_{i_{k}}^{K}\right), \max _{\mathbf{X} \in \mathbf{X}^{\mathbf{I}}}\left(\phi_{i_{j}}^{K} \phi_{i_{k}}^{K}\right)\right]^{-1} \\
\hat{m}_{i}^{S} & =\left[\min _{\mathbf{X} \in \mathbf{X}^{\mathbf{I}}}\left(\phi_{i_{j}}^{M} \phi_{i_{k}}^{M}\right), \max _{\mathbf{X} \in \mathbf{X}^{\mathbf{I}}}\left(\phi_{i_{j}}^{M} \phi_{i_{k}}^{M}\right)\right]^{-1}
\end{aligned}
$$

with $\phi_{\mathbf{i}}^{\mathbf{K}}$ and $\phi_{\mathbf{i}}^{\mathbf{M}}$ the stiffness and mass normalied eigenvectors of the system. At this point, distinction is made between positive and negative modes, referring to the fact that these modal parameter ranges constitute either positive or negative intervals.

step 1.2 The modal envelope FRF is calculated by substituting the ranges of the modal parameters in the deterministic expression of the modal FRF contribution:

$$
\left(F R F_{j k}^{i}\right)^{I}=\frac{1}{\hat{k}_{i}^{S}-\omega^{2} \hat{m}_{i}^{S}}
$$

This is an analytical procedure performed using the interval arithmetic approach. 
step 2 Finally, the total interval FRF is obtained by the summation of the contributions of all considered modes:

$$
F R F_{j k}^{I}=\sum_{i=1}^{n}\left(F R F_{j k}^{i}\right)^{I}
$$

Also this final step is performed using interval arithmetics.

\subsection{Eigenvalue interval correction}

The method as described above can be enhanced based on a graphical interpretation of the modal part of the interval algorithm. For each mode, we consider the domain of modal mass and stiffness pairs that can be achieved by considering the complete range of models defined by the interval uncertainty space $\mathrm{x}^{\mathbf{I}}$ :

$$
\left\langle\hat{k}_{i}, \hat{m}_{i}\right\rangle=\left\{\left(\hat{k}_{i}, \hat{m}_{i}\right) \mid\left(\mathbf{x} \in \mathbf{x}^{\mathbf{I}}\right)\right\}
$$

This domain defines a bounded area in a $\hat{k}_{i}, \hat{m}_{i}$-workspace. The exact bounds of this domain however, are generally unknown. The modal part of the interval algorithm now can be interpreted in this workspace. From the optimisation as described for step 1.1, it is clear that the calculated ranges on the modal parameters $\hat{k}_{i}^{S}$ and $\hat{m}_{i}^{S}$ represent a rectangular approximation of the actual $\left\langle\hat{k}_{i}, \hat{m}_{i}\right\rangle$-domain. Therefore, this method is referred to as the Modal Rectangle (MR) method. Figure 2 shows a general $\left\langle\hat{k}_{i}, \hat{m}_{i}\right\rangle$-domain and its approximation using the MR method.

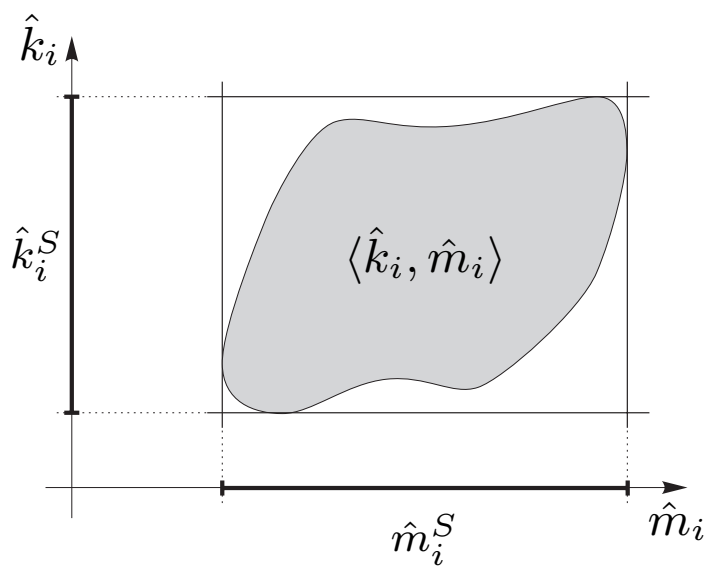

Figure 2: Graphical illustration of a mode's $\left\langle\hat{k}_{i}, \hat{m}_{i}\right\rangle$-domain and its approximation using the modal rectangle method.

The interval arithmetic procedure for the calculation of the modal envelope FRF contributions in step 1.2 can be interpreted in the same graphical domain. The goal in this step is to derive the bounds on the deterministic modal FRF, taking into account that $\hat{k}_{i}$ and $\hat{m}_{i}$ can be anywhere inside their intervals. By considering the modal FRF contribution as defined in equation (39) as an analytical function of $\hat{k}_{i}$ and $\hat{m}_{i}$, the bounds on this function over the modal rectangle have to be determined. It has been shown that this can be done analytically for the complete frequency domain, by considering only the function evaluations at the upper left and the lower right corner points of the rectangle.

Based on these observations, it becomes clear that the calculation based on the modal rectangle introduces conservatism in the procedure if the actual $\left\langle\hat{k}_{i}, \hat{m}_{i}\right\rangle$-domain differs strongly 
from the approximate rectangle. This is often the case, as these parameters are actually coupled through the global system and generally show a high degree of correlation. The proposed enhancement therefore focuses on an improved approximation of the $\left\langle\hat{k}_{i}, \hat{m}_{i}\right\rangle$-domain. This can be achieved by using information on the eigenvalue ranges, which can be obtained using an additional eigenvalue optimisation step in the modal part of the algorithm. It is clear that an eigenvalue interval $\lambda_{i}^{I}$ introduces an extra restriction on the quotient of possible combinations of the modal parameters. This restriction is mathematically expressed as:

$$
\underline{\lambda_{i}} \leq \frac{\hat{k}_{i}}{\hat{m}_{i}} \leq \overline{\lambda_{i}}
$$

Graphically, the eigenvalue bounds represent the lines through the origin of the $\hat{k}_{i}, \hat{m}_{i}$-space which are tangent to the actual $\left\langle\hat{k}_{i}, \hat{m}_{i}\right\rangle$-domain. These lines are extra delimiters for the $\left\langle\hat{k}_{i}, \hat{m}_{i}\right\rangle$ domain approximation, and therefore give rise to an improved $\left\langle\hat{k}_{i}, \hat{m}_{i}\right\rangle$-domain approximation as illustrated in figure 3. This domain is referred to as the Modal Rectangle with Eigenvalue interval correction (MRE).

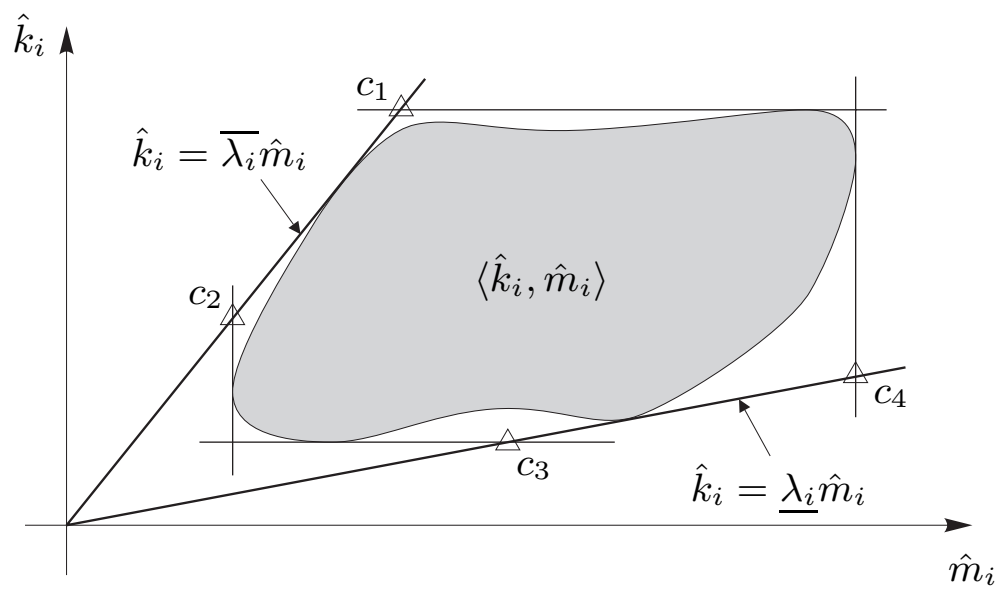

Figure 3: Effect of the introduction of the exact eigenvalue interval in the $\left\langle\hat{k}_{i}, \hat{m}_{i}\right\rangle$-domain approximation of a positive mode.

It has been shown that the conservatism in the modal envelope FRF contributions derived in step 1.2 is substantially reduced by considering the MRE domain instead of the MR domain as area of possible modal parameter pairs. The corresponding modal envelope FRF contributions are determined analytically by calculating the deterministic modal FRFs at the vertex points of the MRE-domain (indicated with $c_{i}, i=1 \ldots 4$ in figure 3). This yields:

$$
\left(F R F_{j k}^{i}\right)^{I}= \begin{cases}{\left[\frac{\overline{\lambda_{i}}}{\overline{\hat{k}_{i}}\left(\overline{\lambda_{i}}-\omega^{2}\right)}, \frac{\underline{\lambda_{i}}}{\underline{\hat{k}_{i}}\left(\underline{\lambda_{i}}-\omega^{2}\right)}\right]} & \text { for } \omega^{2} \leq \underline{\lambda_{i}} \\ {\left[\frac{\overline{\lambda_{i}}}{\overline{\hat{k}_{i}}\left(\overline{\lambda_{i}}-\omega^{2}\right)}, \frac{1}{\overline{\hat{m}_{i}}\left(\underline{\lambda_{i}}-\omega^{2}\right)}\right]} & \text { for } \underline{\lambda_{i}}<\omega^{2}<\overline{\lambda_{i}} \\ {\left[\frac{1}{\hat{\hat{m}}_{i}\left(\overline{\lambda_{i}}-\omega^{2}\right)}, \frac{1}{\overline{\hat{m}_{i}}\left(\underline{\lambda_{i}}-\omega^{2}\right)}\right]} & \text { for } \overline{\lambda_{i}} \leq \omega^{2}\end{cases}
$$


for positive modes, and:

$$
\left(F R F_{j k}^{i}\right)^{I}= \begin{cases}{\left[\frac{\underline{\lambda_{i}}}{\overline{\hat{k}_{i}}\left(\underline{\lambda_{i}}-\omega^{2}\right)}, \frac{\overline{\lambda_{i}}}{\underline{\hat{k}_{i}}\left(\overline{\lambda_{i}}-\omega^{2}\right)}\right]} & \text { for } \omega^{2} \leq \underline{\lambda_{i}} \\ \left.\frac{1}{\underline{\hat{m}_{i}}\left(\underline{\lambda_{i}}-\omega^{2}\right)}, \frac{\overline{\lambda_{i}}}{\underline{\hat{k}_{i}}\left(\overline{\lambda_{i}}-\omega^{2}\right)}\right] & \text { for } \underline{\lambda_{i}}<\omega^{2}<\overline{\lambda_{i}} \\ {\left[\frac{1}{\underline{\hat{m}_{i}}\left(\underline{\lambda_{i}}-\omega^{2}\right)}, \frac{1}{\overline{\hat{m}_{i}}\left(\overline{\lambda_{i}}-\omega^{2}\right)}\right]} & \text { for } \overline{\lambda_{i}} \leq \omega^{2}\end{cases}
$$

for negative modes. It has been shown that this enhancement leads to a close and guaranteed outer approximation of the actual modal envelope FRF contribution [1].

\section{CALCULATION OF RESPONSE RANGE SENSITIVITIES}

In the context of the interval sensitivity analysis as described in section 2 , the response range sensitivity with respect to an input parameter $p$ is expressed as:

$$
\delta_{p^{I}}^{F R F^{I}}(\omega)=\frac{\partial\left(\Delta F R F_{j k}(\omega)\right)}{\partial(\Delta p)}
$$

It is clear that for the FRF interval analysis, the interval sensitivities on the dynamic response depend on the frequency. This frequency dependency is of great importance in the interpretation of the response range sensitivities in a dynamic interval $\mathrm{FE}$ analysis.

Using the concept of the lower and upper bound sensitivities introduced in section 2.3, the response range sensitivity is written as:

$$
\begin{aligned}
\delta_{p^{I}}^{F R F_{j k}^{I}}(\omega) & =\frac{1}{2}\left(\frac{\partial \overline{F R F_{j k}(\omega)}}{\partial(\Delta p)}-\frac{\partial F R F_{j k}(\omega)}{\partial(\Delta p)}\right) \\
& =\frac{1}{2}\left(\sum_{i=1}^{n_{\text {modes }}} \frac{\partial \overline{F R F_{j k}^{i}(\omega)}}{\partial(\Delta p)}-\sum_{i=1}^{n_{\text {modes }}} \frac{\partial F R F_{j k}^{i}(\omega)}{\partial(\Delta p)}\right) \\
& =\frac{1}{2}\left(\sum_{i=1}^{n_{\text {modes }}} \overline{\overline{F R F_{j k}^{i}}}(\omega)-\sum_{i=1}^{n_{\text {modes }}} \delta_{p^{I}}^{F R F_{j k}^{i}}(\omega)\right)
\end{aligned}
$$

This means that the calculation of the response range sensitivity comes down to the calculation of the lower and upper bound sensitivities on the modal FRF contributions.

Equations (43) and (44) indicate that the bounds on the modal response contributions are calculated based on the bounds on the modal parameters and eigenfrequencies. Therefore, we can apply the procedure for the calculation of interval sensitivities in the context of interval arithmetic analysis as introduced in section 2.3.2. For a positive mode, this yields:

$$
\delta \frac{F R F_{j k}^{i}}{p^{I}}(\omega)= \begin{cases}\frac{-\overline{\lambda_{i}}\left(\overline{\lambda_{i}}-\omega^{2}\right) \delta_{p^{I}}^{\overline{\hat{k}_{i}}}-\overline{\hat{k}_{i}} \omega^{2} \delta_{p^{I}}^{\overline{\lambda_{i}}}}{{\overline{\hat{k}_{i}}}^{2}\left(\overline{\lambda_{i}}-\omega^{2}\right)^{2}} & \text { for } \omega^{2} \leq \overline{\lambda_{i}} \\ \frac{-\left(\overline{\lambda_{i}}-\omega^{2}\right) \delta \frac{\hat{m}_{i}}{p^{I}}-\hat{m}_{i} \delta_{p^{I}}^{\overline{\lambda_{i}}}}{{\underline{\hat{m}_{i}}}^{2}\left(\overline{\lambda_{i}}-\omega^{2}\right)^{2}} & \text { for } \overline{\lambda_{i}}<\omega^{2}\end{cases}
$$


for the modal response contribution lower bound, and:

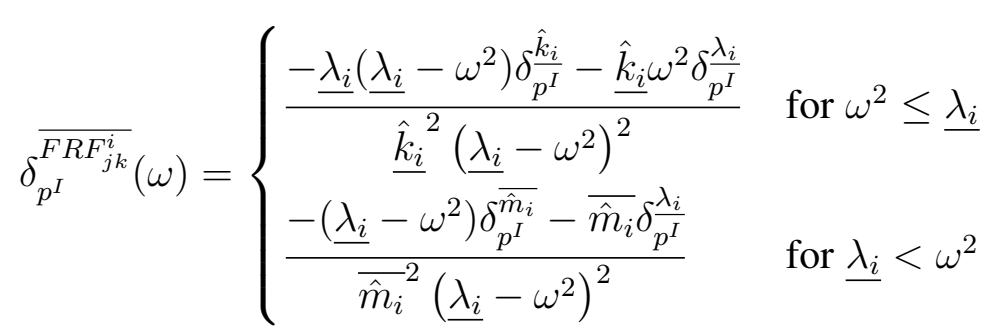

for the modal response contribution upper bound. Similar expressions are found for the modal response contribution lower and upper bound sensitivities of negative modes.

Equations (49) and (50) show that the modal response contribution lower and upper bound sensitivities can be calulcated if the interval sensitivities of the modal parameters of each mode are known. Since the modal parameter intervals are calculated in a global optimisation step (see step 1.1 in section 3), the corresponding interval sensitivities are derived using the procedure described in section 2.3.1. This yields:

$$
\begin{aligned}
& \delta_{p^{I}}^{\overline{\hat{k}_{i}}}=\left|\left(\frac{\partial \overline{\hat{k}_{i}}}{\partial p}\right)_{p^{\hat{k}_{i}}}\right| \quad, \quad \delta_{p^{I}}^{\hat{k}_{i}}=-\left|\left(\frac{\partial \hat{\hat{k}_{i}}}{\partial p}\right)_{p_{\underline{\hat{k}_{i}}}}\right| \\
& \delta_{p^{I}}^{\overline{m_{i}}}=\left|\left(\frac{\partial \overline{\hat{m}_{i}}}{\partial p}\right)_{p^{\hat{m}_{i}}}\right| \quad, \quad \delta_{p^{I}}^{\frac{\hat{m}_{i}}{I}}=-\left|\left(\frac{\partial \hat{m}_{i}}{\partial p}\right)_{p \frac{\hat{m}_{i}}{}}\right| \\
& \delta_{p^{I}}^{\overline{\lambda_{i}}}=\left|\left(\frac{\partial \overline{\lambda_{i}}}{\partial p}\right)_{p^{\lambda_{i}}}\right| \quad, \quad \delta_{p^{I}}^{\frac{\lambda_{i}}{I}}=-\left|\left(\frac{\partial \underline{\lambda_{i}}}{\partial p}\right)_{p_{\underline{i}}^{\lambda_{i}}}\right|
\end{aligned}
$$

In the procedure, these end point gradients are generally available as a part of the optimisation results. Therefore, they are easily recuperated and substituted in equations (49) and (50). Substituting the resulting modal response contribution lower and upper bound sensitivities in equation (48) then finally gives the required response range sensitivity.

\section{APPLICATION}

The calculation of the interval sensitivities in the context of dynamic response analysis is illustrated using a numerical case study of a truck model. The aim of the study is to analyse the influence of uncertain design parameters of the model on the dynamical behaviour of the construction.

\subsection{Nominal model}

The model under consideration is chosen such that it represents the most critical dynamic behaviour of a commercial transport vehicle in the low frequency domain. The model consists of a series of local masses, springs and dampers, and is assembled such that it represents the total truck as a dynamic system, incorporating realistic suspension behaviour. The modal characteristics of this model correspond extremely well with those of a full size truck model. The model however is far more economical from computational point of view, and therefore serves as a reference during initial studies aiming at the prediction of ride comfort of new truck designs.

The reference model is a 13 degrees of freedom system representing a tractor semi-trailer combination, and is illustrated with its mechanical components in figure 4 . Only symmetric 


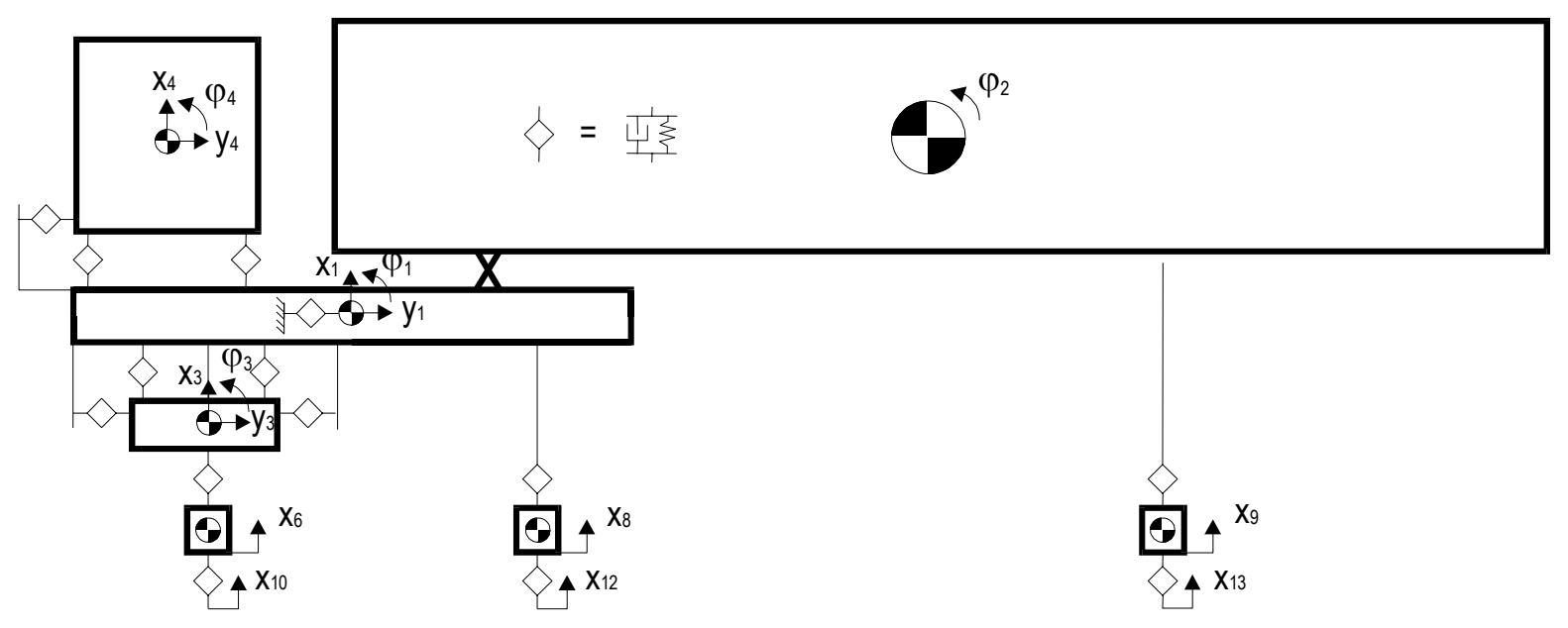

Figure 4: Reference model of the truck vehicle reduced to its principal dynamic behaviour in the low frequency domain

motion is considered, and chassis flexibility is ignored. The provided model has 13 degrees of freedom:

- $x_{4}, y_{4}, \varphi_{4}$ : cabin horizontal, vertical translation, and pitch

- $x_{3}, y_{3}, \varphi_{3}$ : engine horizontal, vertical translation, and pitch

- $x_{1}, y_{1}, \varphi_{1}$ : chassis horizontal, vertical translation, and pitch

- $\varphi_{12}$ : trailer pitch

- $x_{6}, x_{8}, x_{9}$ : vertical translation at wheel axles

The vertical translations at the wheel base, represented by $x_{10}, x_{12}$ and $x_{13}$, are considered as input degrees of freedom where vertical displacements are introduced in the model. In order to do a dynamic analysis in the frequency domain, the model is excited with a harmonic acceleration in these degrees of freedom, representing as such the vertical translation induced by the road roughness at the road-tyre contact. The ride comfort is then analysed based on the transfer function between a base acceleration on the individual tyres and the acceleration in the cabin. The analysis here focuses on the front-most tyre.

In order to calculate the frequency response function between the road acceleration and the cabin acceleration, base excitation is modelled at the tyre-road interface points using the large mass modelling concept. For this purpose, the model is extended with an additional large point mass rigidly connected to the structure at the input degrees of freedom. The mass of the added element equals $10^{6}$ times the total mass of the original structure. A harmonic excitation force on the large mass serves as input for the dynamic analysis. The resulting force-acceleration response function can then be easily transformed to the required acceleration-acceleration form by multplying the final response function with the total mass of the extended model.

\subsection{Interval sensitivity analysis}

A case with three uncertain parameters is examined. These represent the uncertainty on the mass properties of the chassis, trailer and cabin $\left(m_{1}, m_{2}\right.$ and $\left.m_{4}\right)$. The interval analysis is 


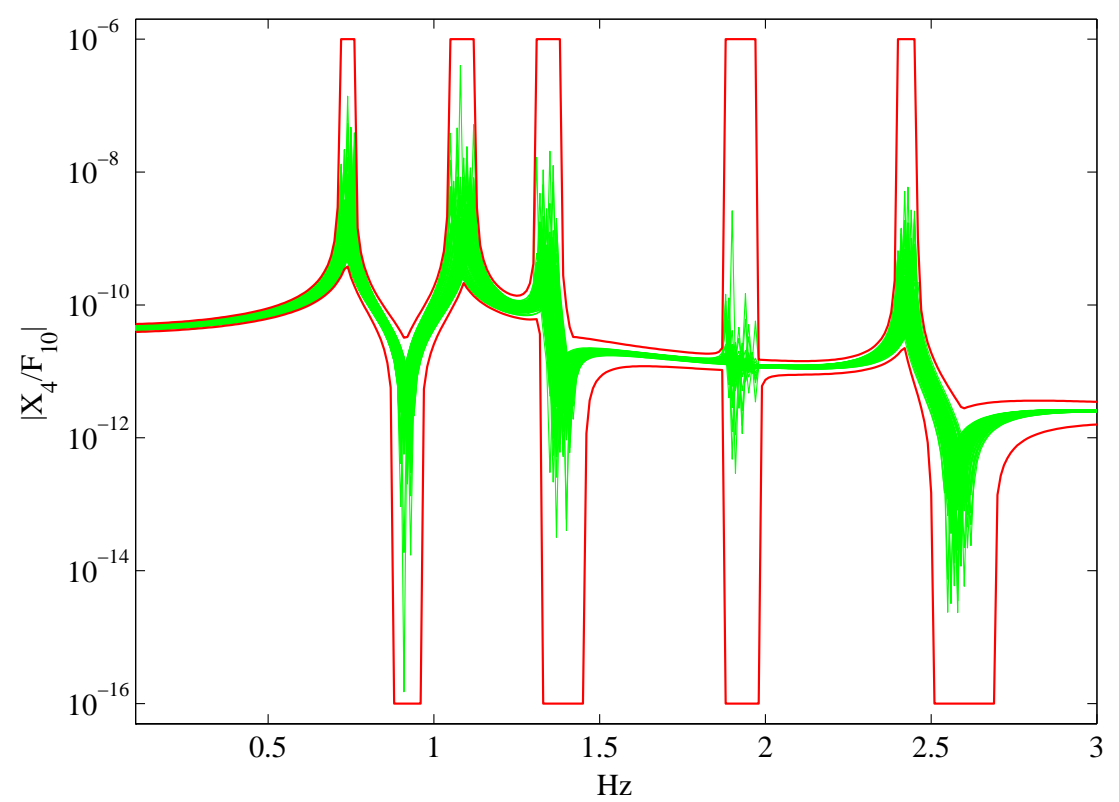

Figure 5: Envelope frequency response function between the front tyre and the cabin vertical acceleration considering interval uncertainties for the 3 uncertain mass properties (red: interval bounds; green: Monte Carlo samples)

performed with interval uncertainties ranging over the nominal values for these masses $+/$ $10 \%$. The MRE methodology is applied, resulting in the interval FRF as shown in figure 5.

The interval sensitivities on the dynamic response range have been calculated using the formulas of the previous section. Figure 6 shows the evolution of the interval sensitivities in the frequency domain. In order to enable a comparison of the relative importance of the interval sensitivities of each individual parameter, the results have been scaled such that the sum of the interval sensitivities at each frequency line equals one.

It can be clearly observed that the cabin mass uncertainty has a dominant influence on the response uncertainty. This is due to the fact that the response level at the cabin centre of mass is taken as output degree of freedom of the observed FRF. The importance of the cabin mass is more pronounced in the lower part of the frequency domain. This stems from the fact that the cabin pitch and bounce modes are located around $1 \mathrm{~Hz}$. It is clear that the mass of the cabin becomes more important in this region of the FRF. Further, a clear impact of the chassis mass uncertainty on the global response uncertainty is visible around $2.5 \mathrm{~Hz}$. This is at the location of the cabin-chassis bounce mode. The fact that the chassis has a dominant role in the uncertainty on the cabin response level leads to the conclusion that efforts on limiting the uncertainty or variance on the chassis mass will add to a more robust design with respect to dynamic response uncertainty at the driver position in the cabin.

\section{ACKNOWLEDGEMENT}

The truck model was kindly made available by DAF TRUCKS HOLLAND.

\section{CONCLUSIONS}

This paper introduces the concept of interval sensitivities, applicable in the context of general numerical interval analysis. This concept enables the quantification of the relative effect of individual uncertain input parameters on the ouput range obtained by the interval analysis. A high interval sensitivity of the output with respect to a specific uncertain input parameter indicates a 


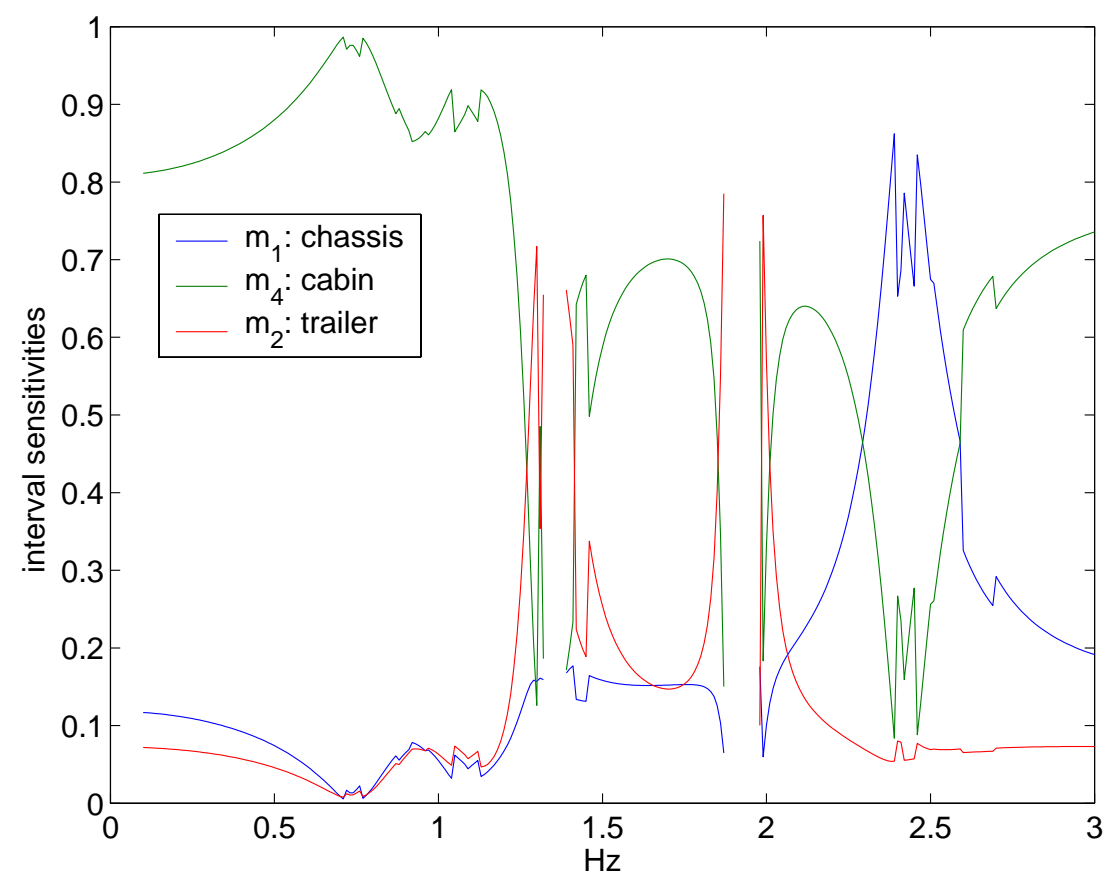

Figure 6: Evolution of the interval sensitivities of the dynamic response width for the frequency response function between the front tyre and the cabin vertical acceleration with respect to the uncertain mass properties of the model

strong influence of this parameter on the obtained uncertainty on the result of the analysis. As such, this concept can be of great value in the practical application of interval analysis.

This paper also shows how the concept of interval sensitivities can be applied to the hybrid dynamic response range calculation procedure in the framework of interval finite element analysis. The response range sensitivities are obtained as analytic functions of end point gradients of the model parameters, which follow directly from the optimisation part of this algorithm. Consequently, the calculation of the response range sensitivities is computationally not more expensive than the original interval FRF analysis.

Finally, the paper shows the application of the response range sensitivity analysis on a simplified but realistic truck model. The interval sensitivity concept is shown to be very appropriate for the analysis of the effect of input interval uncertainties on the resulting uncertainty on the output side of the problem, as it helps to identify the most crucial uncertainties in the defined problem.

\section{REFERENCES}

[1] Moens, D. and Vandepitte, D., "An Interval Finite Element Approach for the Calculation of Envelope Frequency Response Functions," International Journal for Numerical Methods in Engineering, Vol. 61, No. 14, 2004, pp. 2480-2507.

[2] Moens, D. and Vandepitte, D., "Application of the Fuzzy Finite Element Method to Spacecraft Design," Proceedings of the International Conference on Spacecraft, Structures and Mechanical Testing, ESA SP-581, 2005.

[3] De Gersem, H., Moens, D., and Vandepitte, D., "A Fuzzy Finite Element Procedure for the Calculation of Uncertain Frequency Response Functions of Damped Structures: Part 2 - 
Numerical Case Studies," Journal of Sound and Vibration, Vol. 288, No. 2, 2004, pp. 463486.

[4] Moens, D. and Vandepitte, D., "A Survey of Non-Probabilistic Uncertainty Treatment in Finite Element Analysis," Computer Methods in Applied Mechanics and Engineering, Vol. 194, No. 14-16, 2005, pp. 1527-1555. 